\title{
Minimize the Energy Consumption and Maximize the Throughput for Wireless Sensor Network using Routing Algorithm
}

\author{
Sunita \\ M.E Student (ECE) \\ Gurdaspur (pb.)
}

\author{
O.S Khanna \\ Associate Professor (ECE) \\ Chandigarh
}

\author{
Amandeep Kaur \\ M.E Student (ECE) \\ Mansa (pb.)
}

\begin{abstract}
The major challenge in wireless sensor networks is to decrease energy consumption and to increase the throughput of sensor node to enhance the lifetime of sensor network. The routing protocols have great impact on the lifetime of sensor network. Mainly the data packet delivery ratio and average energy consumption are examined as the performance metrics. Simulation results show that proposed algorithm performs better than EQSR (Energy Efficient and QOS aware multipath routing) and comparison is performed in term of data packet delivery ratio and energy consumption.
\end{abstract}

\section{General Terms}

Wireless sensor network, Two-pass Algorithm, Energy Efficient and QoS multipath routing (EQSR)

\section{Keywords}

WSN, energy efficiency, throughput, multipath routing, Quality of service.

\section{INTRODUCTION}

A sensor network is a framework which comprises of sensing, computation and communication into a single tiny device. Wireless sensor networks are used in various applications to monitor the environment conditions such as humidity and pressure, temperature, noise levels, mechanical stress; lightning conditions at different locations [1]. Recent advancement in technology permits the development of low-cost, low power, and multifunctional sensor devices. These nodes have capability to sense, compute and processing the data [2]. A sensor network consists of huge number of small sensors nodes, whose aim in 'field of interest' region to observe a single or multiple phenomena [3]. These nodes have limited battery power. Data routed from source to destination may follow many possible routes. Suppose source node generates data and is delivered to destination node. If the node has sufficient battery power only then it will transmit data to the distance node. If the node lies in its transmission range, then only data can transmit between two nodes without any failure. If the node is situated far away from the source node, where data is transferred, then large battery power is required to transmit the data. After few transmissions, a stage will reached when a node eliminated from the network path because it reached to its threshold battery level. A situation will be reached when no node will be available for transmission of data and the overall lifetime of a network decrease [4]. For enhancing the lifetime of network, there should be balance between energy consumption used by nodes and their energy reserved.

Power-Aware Routing Algorithm based on Mobile Agents (PARAMA) to reduce the energy consumption is proposed in [5]. Using this approach, the battery consumption is reduced but only applicable to less network traffic. A new hierarchical routing protocol EL-LEACH (Energy and Load balance LEACH) is proposed in [6] which achieve better energy consumption but the throughput is decreased and delay is increased. Jalel Ben-Othman et.al [7] proposed Energy efficient and QoS based routing protocol (EQSR) for wireless sensor networks which results energy savings, low delay, high packet delivery. A conditional max min battery capacity routing (CMMBCR) scheme is proposed in [8] that chooses the shortest path if all nodes in the route have enough battery capacity. These routes will be ignored if the battery capacity of nodes goes below a threshold value. So the battery capacity of those nodes will not be terminate completely and the lifetime of the network is increased. Many energy savings schemes in wireless sensor network are discussed in [9] .They classify three techniques duty cycling, data-driven and mobility to minimize the power consumption. Distributed energy balanced routing is proposed [10]. For the transmission of data, this algorithm uses the energy balance path. Firstly, find the energy cost of all the paths between source and destination nodes and then chooses the energy efficient path for data transmission. A probabilistic model based on Petri nets is discussed in [11] to find the energy consumption of a node in wireless sensor network. But it requires the long simulation time

The remaining paper is organized as follows: In section 2, Architecture of wsn is discussed .In section 3, EQSR routing protocol is described. In section 4, proposed work is explained. In section 5, simulation results and discussion is held. And, in section 6 , paper is concluded.

\section{ARCHITECTURE OF WSN}

Sensor nodes consist of small or large number of nodes in the sensor network. These nodes have different types of modules such as Sensor Module, Processing Module, Wireless Communication Module and Power Supply Module. To make the sensor operational in wireless sensor network, these modules work together in such environment [12]. Fig 1 
illustrates the architecture of wireless sensor nodes and different modules summarized as:

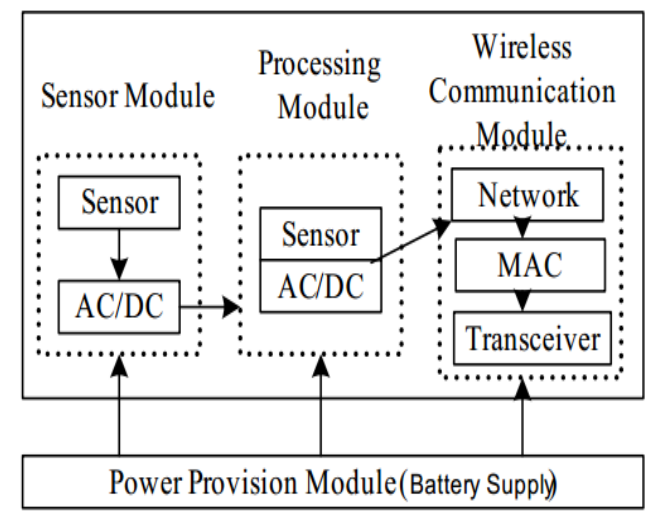

Figure 1 Architecture of a sensor node [12]

Sensor Module consists includes signal sampling, A/D signal conversion and signal modulation. Based on the observed phenomena, sensors produce the analog signals which are converted into digital signals by A/D [12]. Processing Module include the controlling the sensor, communication between protocols and processing the data or information. The digital signals from the sensor module are fed into the processing module that carries the sensing tasks. In Wireless Communication Module, A radio transceiver generates the radio waves and also connects the nodes to the network. The power supply module consists of power unit (battery) which is supported by power scavenging unit such as solar cells and also related to the manufacturer [13].

\section{EQSR PROTOCOL}

Sometimes to satisfy the QoS metrics, such as delay, energy and bandwidth, some routing protocols are more prone to route failures [14] .To maintain the routing table and QoS metrics, the sensor nodes have to face the overhead problems. The protocols are divided into two categories: Multipath based protocols and QoS-based protocols multipath based protocols. Multipath routing provides much better overall network performance by allowing better sharing of the available network resources and more prone to node failures. According to the type of data, QoS based protocol route the traffic. Maintain the balance between energy consumption and data quality is the main task of network. The routing algorithms like short path routing algorithm that calculate the minimum cost length to find the shortest path from source to destination node. In that case, the source nodes constantly generate the packets and routes the packets through same shortest path. Sometimes the minimum cost length that calculated is not accurate. Constantly generation of packets that overloaded and leads to congestion. It will increase the delay and packet losses, which in turns will cause retransmission of packets. And that increase the energy consumption. So in proposed work, this paper suggests the Two pass algorithm in which we calculate minimum cost length accurately through which the packets are routed.

\section{PROPOSED WORK}

The critical issues in wireless sensor network are energy consumption and throughput. So, in proposed paper; it focuses on energy consumption and throughput of the network. For the successful delivery of packets to their destination or we can say throughput, the selection of the routing strategies are most important issue. The routing strategy that applied in such networks should confirm that the energy consumption will decrease and hence the lifetime of such network will be increased [15]. The base paper uses the protocol EQSR (Energy Efficient and QoS multipath aware routing) to increase the lifetime of network. The main drawback of base paper was there was increase in the energy consumption in cost of end-toend delay. The end-to-end delay was minimized but energy consumption was increased in real time application. But, in this proposed paper modified the protocol EQSR, in which we implement the two pass algorithm in EQSR routing algorithm. So the energy consumption is decreased and throughput is increased. The two pass algorithm is as followed

\section{Two pass algorithm}

Assume $\mathrm{n}$ be the number of nodes in network and $\mathrm{N}_{\mathrm{s}}$ be the sensor array representing the sensor nodes.

Let XLOC, YLOC and array representing location of nodes in network. If $\mathrm{L} \& \mathrm{~B}$ be the dimension of network service area and the sensors are randomly deployed in the network of dimensions $1000 * 1000$.

$$
\text { Area of network }=\mathrm{L} * \mathrm{~B}=1000 * 1000 \text {. }
$$

Let XLOCs be the X-coordinate for the source node. In simple words, it is the first node that starts sensing data, aggregate the data and buffer the data. It is the first node that tries to find the route to send the data. Suppose, it is the temperature sensor and it has a reading of $25^{\circ} \mathrm{C}$. Now, it wants to send the information of $25^{\circ} \mathrm{C}$ of particular location to monitoring station and the monitoring station is very far and beyond the transmission range of its sensor.

Let $\mathrm{T}$ be the transmission range of sensor node. If $\mathrm{A}$ is source and $\mathrm{B}$ is destination, then if $\operatorname{direct} \mathrm{A}$ sends data to $\mathrm{B}$, but $\mathrm{B}$ is very far or away from its transmission range.

Let's transmission range of node $=25 \mathrm{~m}$

\section{If distance $<=$ transmission range}

Send data

else

Wait for other which helps in sending data

For this problem we add three forwarding nodes $\mathrm{L}, \mathrm{M}$ and $\mathrm{N}$ in the mid of A and B that helps in forwarding data and also maintains the routing table, R. Now problem is that source has three options for sending data either through $\mathrm{L}$ or $\mathrm{M}$ or $\mathrm{N}$. But the source doesn't know the optimum path for sending data. So firstly, 
- It checks energy, if energy is same for all forward nodes $(\mathrm{L}, \mathrm{M}$, and $\mathrm{N}$ ).then which optimum path will be taken. That question arises.

- Then, it checks payload or data, if sending same data suppose 10kj data for all forwarding nodes, again same problem.

- Then, It Check for buffer .but if forwarding nodes L, M, N has same memory then same problem.

- Then, it checks for SNR. If forwarding nodes L, M, N has same link quality function. Then same problem comes.

Routing table that maintain the updates

$$
\begin{aligned}
& \mathrm{R}=\mathrm{x}_{1}=\text { residual energy } \\
& \mathrm{R}_{2}=\mathrm{x}_{2}=\text { payload } \\
& \mathrm{R}_{3}=\mathrm{x}_{3}=\text { free buffer } \\
& \mathrm{R}_{4}=\mathrm{x}_{4}=\mathrm{SNR}
\end{aligned}
$$

$\mathrm{R}_{1}, \mathrm{R}_{2}, \mathrm{R}_{3}$ and $\mathrm{R}_{4}$ are the routing tables

Then we find the cost function,

$$
f(c)=x_{1}+x_{2}+x_{3}+x_{4}+c .
$$

Total cost $\mathrm{J}_{\text {total }}$ is the sum of individuals link costs $\mathrm{f}(\mathrm{c})$.

$$
\mathrm{J}_{\text {total }}=\sum_{i}^{n-1} f(c) i
$$

Where $\mathrm{n}$ is set of nodes.

Sometimes mean or cost function is not stable or accurate, when we calculate. Suppose it be 6.5 or 6.7 and it is low cost function, then we follow the shortest path which has low cost function. Data is routed through same path but after sometime it comes to know that the mean or cost function we calculate is not exact. It increases the delay and causes the retransmission of data. That increases the energy consumption. Sometimes the cost function or mean that not constant and is varied time to time or in language of wireless communication, signal sometimes varied to left or right. So in proposed algorithm we go for variance.

By use of variance, we get the stable point, but sometimes signal or mean again propagates and so we again go for second variance to get the optimum cost function and hence optimum shortest path. That's why it termed as two pass algorithm. By using this algorithm, there is no chances to worry about the exact cost function if we get the exact cost function, then data will routed through same shortest path and no delay occur and no chances of retransmission of data, because the data delivered successfully through that shortest path. So, no energy will be wasted to decide which shortest path we follow. As a result energy consumption decreases and more and more packets send, as a result throughput increases. The pseudo code of two pass algorithm is as followed:

Using this algorithm, for variance firstly we compute sample mean and then compute sum of squares from mean as follow

\section{$\underline{\text { Algorithm }}$}

Two_pass_algorthim(data ):

$\mathrm{i}=0$

Add $1=0$

Add 2 $=0$

for $\mathrm{i}$ in data

$\mathrm{i}=\mathrm{i}+1$

Add $1=$ Add $1+\mathrm{i}$

Mean $=\frac{\operatorname{Add} 1}{i}$

For $\mathrm{i}$ in data

Add $2=$ Add $2+(\mathrm{x}-\mathrm{mean}) *(\mathrm{x}-$ mean $)$

Variance $=\frac{\operatorname{Add} 2}{i-1}$

Return variance;

\section{Results and discussion}

In this section, the aim of this simulation study is to analyze the performance of proposed routing protocol and compared with the existing routing protocol. The simulations have been performed using Network Simulator 2 version 2.34, a software that provides scalable simulations of Wireless Networks and an open source software that used in studying the dynamic nature of communication networks, and simulations behaviors of wired as well as wireless network functions and protocols (routing algorithms, TCP,UDP)and object-oriented network simulator [14]. NS 2, is a discrete-event driven simulation tool that contains two set of languages; namely $\mathrm{C}++$ and OTCL. $\mathrm{C}++$ is used for the creation of objects because of speed and efficiency. OTcl is used as a front-end to setup the simulator, configure objects and schedule events because of its ease of use [16]. Our simulation network consists of 200 sensor nodes are randomly deployed in a field of $1000 \mathrm{~m} * 1000 \mathrm{~m}$. All nodes have same transmission range of $25 \mathrm{~m}$. The simulation parameters are shown in Table 1.

Table 1 Simulation parameters

\begin{tabular}{|l|l|}
\hline Network field & $1000 \mathrm{~m} \times 1000 \mathrm{~m}$ \\
\hline Propagation model & Two ray ground \\
\hline Number of sensors & 200 \\
\hline $\begin{array}{l}\text { Number of sinks/number } \\
\text { of sources }\end{array}$ & $1 / 1$ \\
\hline Transmission range & $25 \mathrm{~m}$ \\
\hline $\begin{array}{l}\text { Packet size (data }+ \\
\text { overhead) }\end{array}$ & 1024 bytes \\
\hline Sub-packet size & 256 bytes \\
\hline
\end{tabular}




\begin{tabular}{|l|l|}
\hline Transmit power & $15 \mathrm{~mW}$ \\
\hline Receive power & $13 \mathrm{~mW}$ \\
\hline Idle power & $12 \mathrm{~mW}$ \\
\hline Initial battery power & $100 \mathrm{j}$ \\
\hline MAC layer & IEEE 802.11 \\
\hline Max buffer size & 256 K-bytes \\
\hline Simulation time & $400 \mathrm{~s}$ \\
\hline
\end{tabular}

We examined the performance of modified EQSR protocol. The two metrics energy consumption and delivery ratio are evaluated. The average consumption is the average of energy consumed in sending the data from source node to destination. The delivery ratio is defined as the ratio between the number of packets sent and number of packets at destination. It describes the percentage of packets that reached at destination [17].

During the simulation, the values obtained for Modified EQSR in terms of energy consumption and packet delivery ratio and the existing values of EQSR are listed in Table 2 and Table 3.

Table 2 Energy consumption of EQSR and Modified EQSR

\begin{tabular}{|c|c|c|}
\hline Packets/sec & EQSR & $\begin{array}{r}\text { Modified } \\
\text { EQSR }\end{array}$ \\
\hline 10 & 0.028 & 0.00 \\
\hline 20 & 0.034 & 0.00 \\
\hline 30 & 0.039 & 0.00 \\
\hline 40 & 0.041 & 0.00 \\
\hline 50 & 0.044 & 0.01 \\
\hline 60 & 0.046 & 0.01 \\
\hline 70 & 0.05 & 0.01 \\
\hline 80 & 0.055 & 0.01 \\
\hline 90 & 0.059 & 0.02 \\
\hline 100 & 0.062 & 0.02 \\
\hline
\end{tabular}

Table 3 Packet Delivery ratio of EQSR and Modified EQSR

\begin{tabular}{|c|c|c|}
\hline Packets/sec & EQSR & $\begin{array}{c}\text { Modified } \\
\text { EQSR }\end{array}$ \\
\hline 10 & 1.00 & 1.00 \\
\hline 20 & 0.98 & 1.00 \\
\hline 30 & 0.97 & 1.00 \\
\hline 40 & 0.96 & 0.99 \\
\hline 50 & 0.95 & 0.99 \\
\hline 60 & 0.94 & 0.99 \\
\hline
\end{tabular}

\begin{tabular}{|l|l|l|}
\hline 70 & 0.93 & 0.99 \\
\hline 80 & 0.92 & 0.99 \\
\hline 90 & 0.91 & 0.99 \\
\hline 100 & 0.90 & 0.98 \\
\hline
\end{tabular}

From Figure 2 , it is noticed that from coordinates $(0,0)$ to $(90,0.06)$ of EQSR , the values goes on increasing it means that the energy consumption increases due to follow the wrong shortest path, whereas in case of Modified EQSR, the values from coordinates $(0,0)$ to $(40,0)$ are constant and then from coordinates $(40,0)$ to $(90,0.02)$, the values increases. But these values are decreased as compared to EQSR values, it tells that using the two pass algorithm, the data is routed through accurate optimum path. From Figure 3, it shown that in case of EQSR, the values of packet delivery ratio from coordinates (10, $1)$ to $(100,0.90)$ are decreases. It is because of wrong optimum

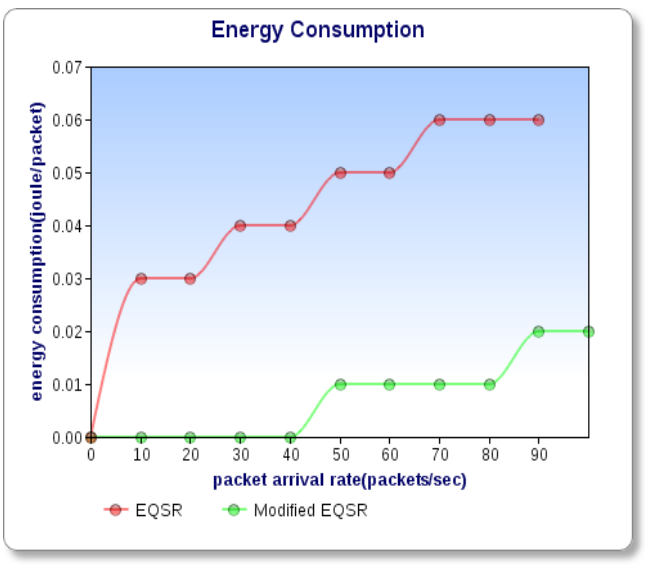

Figure 2 Energy consumption vs packet arrival rate (packets/sec)

path. But in case of modified EQSR, the values of packet delivery ratio are constant from coordinates $(10,1)$ to $(30,1)$, and from co-ordinates $(30,1)$ to $(100,0.98)$, the values slightly decreases, but the packet delivery is more in modified EQSR as compared toEQSR.

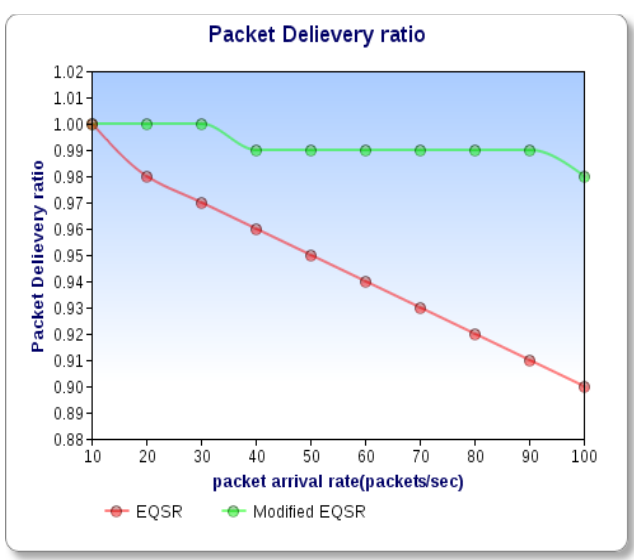

Figure 3 Packet Delivery ratio vs packet arrival rate (packets/sec) 


\section{CONCLUSION}

In this paper, we have presented modified EQSR protocol; An Energy efficient and quality of service aware multi-path routing protocol modified. The performance of modified EQSR is analyzed using NS2 simulator. After analysis, it outcomes that modified EQSR perform better than EQSR in terms of energy consumption and packet delivery ratio. As a future work, due to verifying cost function twice to get the best optimum path, the average delay increases. Future work can be done on the average delay.

\section{REFERENCES}

[1] F. Akyildiz, Weilian Su, Yogesh Sankarasubramaniam, and Erdal Cayirci, "A Survey on Sensor Networks," IEEE Journals \& Magazines on Communication Magazine, vol. 40, pp. 102-114, 2002.

[2] Ivan Stojmenovic and Stephan Olariu, "Data-Centric Protocols for Wireless Sensor Networks," John Wiley \& Sons, 2005

[3] karkvandi,H.R.;Pencht,E.;Yadid-Pencht,O., "Effective Lifetime-Aware Routing in Wireless Sensor Networks," IEEE Journals on Sensors, vol. 11, pp. 3359-3367, 2011

[4] Praveen Kaushik ; Jyoti Singhai, "Energy Efficient Routing Algorithm for Maximizing the Minimum Lifetime of Wireless Sensor Network: A Review" , International Journal of Ad hoc, Sensor \& Ubiquitous Computing (IJASUC), vol.2, no.2, 2011

[5] Yi Zhang; Li Feng; Wei Chen; Lina Zhu, "Power-Aware Routing Algorithm based on Mobile Agents (PARAMA) in Mobile Ad Hoc Networks", International Conference on Embedded Software and Systems Symposia (ICESS), pp. $312-317,2008$.

[6] Thu Ngo Quyn , Kieu-Ha Phun, Hoan Vu Quoc, "Improvement of Energy Consumption and Load Balance for LEACH in Wireless Sensors Networks", International conference on ICT Convergence, pp. 583-588, 2012.

[7] Jalel Ben-Othman, Bashir Yahya, "Energy efficient and QoS based routing protocol for wireless sensor network", IEEE Journals of parallel and Distributed computing, vol 70, pp. 849-857, 2010
[8] Toh C. K., "Maximum battery life Routing to Support Ubiquitous Mobile Computing in Wireless $\mathrm{Ad} \mathrm{Hoc}$ Networks", IEEE communication magazine, pp. 138-147, 2011.

[9] G. Anastasi, M. Conti, M. D. Francesco, A. Passarella "Energy conservation in wireless sensor networks: A survey", IEEE Symposium on Ad Hoc Networks, vol. 7, pp. $537-568,2009$

[10] C-S Ok, S. Lee, P. Mitra and S. Kumara, "Distributed Energy Balanced Routing for Wireless Sensor Networks", IEEE International Conference on Computer \& Industrial Engineering, vol. 57, pp. 125-135, 2009.

[11] Ali Shareef, Yifeng Zhu, "Energy Modeling of Wireless Sensor Nodes Based on Petri Nets", International Conference on Parallel Processing, pp. $101-110,2010$.

[12] I.F. Akyildiz, W. Su*, Y. Sankarasubramaniam, E. Cayirci "Wireless sensor networks: a survey", IEEE Communication Magazine, pp. 393-422, 2002.

[13] Nikolaos A. Pantazis, Dimitrios D. Vergados, "A Survey on Power Control Issues in Wireless Sensor Networks," IEEE Communications Surveys, vol. 9, pp. 86-107, 2007.

[14] Teerawat Issariyakul, Ekram Hossain, "Introduction to Network Simulator NS2", Springer Science and Business Media, LLC, 2009.

[15] Baiping Li; Xiaoqin Zhang, "Research of development in wireless sensor network routing protocols based on NS2" International Conference on Electronic and Mechanical Engineering and Information Technology (EMEIT), vol. 4, pp. 1913-1916, 2011.

[16] Anil Kumar Sharma, Neha Bhatia, "Behavioral Study of MANET Routing Protocols by using NS-2", International Journal of Computational Engineering \& Management(IJCEM), vol. 12, pp. 2230-7893, 2011.

[17] Nikolaos A. Pantazis, Stefanos A. Nikolidakis and Dimitrios D. Vergados, "Energy-Efficient Routing Protocols in Wireless Sensor Networks: A Survey," IEEE Communications Surveys \& Tutorial, vol 3, pp. 1-41, 2012. 